\title{
Valeur de conservation de la Réserve Naturelle Volontaire (RNV) de la Dodo, Sud-Ouest de la Côte d'Ivoire (Afrique de l'Ouest)
}

\author{
Yao Jean-Clovis KOUADIO ${ }^{1 *}$, Keagnon Serge Pacome SOIRET ${ }^{2,3}$, \\ Wokapeu Blaise KPAN ${ }^{1,3}$, N'Guessan Olivier YAO ${ }^{1}$, Kouakou Edouard N'GUESSAN ${ }^{1}$, \\ Kouassi Philippe KOUASSI ${ }^{2}$ et Peñate José GOMEZ ${ }^{4}$
}

${ }^{1}$ Laboratoire de Botanique, UFR Biosciences, Université Félix HOUPHOUËT-BOIGNY, 22 BP 582 Abidjan

22, Côte d'Ivoire.

${ }^{2}$ Laboratoire de Zoologie et Biologie Animale, UFR Biosciences, Université Félix HOUPHOUËT-BOIGNY, 22 BP 582 Abidjan 22, Côte d'Ivoire.

${ }^{3}$ Centre Suisse de Recherches Scientifiques en Côte d'Ivoire (CSRS), Adiopodoumé, 01 BP 1303 Abidjan 01, Côte d'Ivoire.

${ }^{4}$ Conservation des Espèces Marines de Côte d'Ivoire, 06 BP 1412 Abidjan 06, Côte d'Ivoire.

*Auteur correspondant ; E-mail: jeanclovis08@gmail.com; Tel: (+225) 08050892

\section{REMERCIEMENTS}

Le financement de ce travail a été assuré par the Global Wildlife Conservation (GWC) et la Margot Marsh Biodiversity Foundation.

\section{RESUME}

En Côte d'Ivoire, le patrimoine forestier en milieu rural est confronté à une reconversion considérable en espace agricole. Cette situation conduit à la dégradation des habitats et à une perte croissante de la biodiversité. Pour inverser la tendance l'Etat sensibilise à la création de Réserves Naturelles Volontaires. Cependant, cette stratégie ne pourrait avoir l'assentiment de tous si les bienfaits de la diversité biologique en milieu rural ne sont pas développés et connus de tous. La présente étude se propose d'évaluer la valeur de conservation de la Réserve Naturelle Volontaire (RNV) de la Dodo. Pour y arriver, la méthodologie employée a porté sur l'inventaire de surface combiné à l'inventaire itinérant. Les différents types de végétation rencontrés sont les jachères, les savanes incluses, les forêts denses, les forêts secondaires et les forêts galeries. Il a été inventorié 242 espèces reparties 193 genres appartenant à 70 familles. Les familles les plus diversifiées par ordre d'importance sont les Euphorbiaceae, les Rubiaceae, les Caesalpiniaceae, les Annonaceae, les Apocynaceae. Les espèces de la région Guinéo-Congolaise (GC) sont les plus nombreuses. On dénombre vingt et une (21) espèces endémiques et 14 espèces rares et/ou menacées d'extinction. L'abondance des espèces à statut particulier et la présence d'habitats particuliers confèrent à la RNV les valeurs HVC1 et HVC3.

(C) 2018 International Formulae Group. All rights reserved.

Mots clés: Réserve Naturelle Volontaire, conservation, Sud-Ouest, Côte d'Ivoire. 


\title{
Conservation value of the Voluntary Nature Reserve (RNV) of the Dodo, South-Western, Côte d'Ivoire (West Africa)
}

\begin{abstract}
In Côte d'Ivoire, the forest heritage in rural areas is facing a considerable reconversion in agricultural space. This situation leads to habitat degradation and increasing loss of biodiversity. To reverse the trend, the State is sensitizing to the creation of Voluntary Natural Reserves. However, this strategy could not be universally accepted if the benefits of biological diversity in rural areas are not developed and known to all. The present study proposes to evaluate the conservation value of the Voluntary Natural Reserve (RNV) of Dodo. To achieve this, the methodology used was for surface inventory combined with itinerant inventory. The different types of vegetation encountered are fallows, savannahs included, dense forests, secondary forests and gallery forests. It has been inventoried 242 species distributed 193 genera belonging to 70 families. The most diverse families in order of importance are Euphorbiaceae, Rubiaceae, Caesalpiniaceae, Annonaceae, Apocynaceae. The species of the Guineo-Congolese (GC) region are the most numerous. There are twenty-one (21) endemic species and 14 rare and / or endangered species. The abundance of species with special status and the presence of particular habitats give the RNV HVC1 and HVC3 values.
\end{abstract}

(C) 2018 International Formulae Group. All rights reserved.

Keywords: Voluntary nature reserve, conservation, south-western, Côte d'Ivoire.

\section{INTRODUCTION}

Les écosystèmes et les habitats font l'objet de protection par plusieurs instruments juridiques en Côte d'Ivoire. On peut citer, en autres, la loi n²014-427 du 14 juillet 2014 portant code forestier, la loi $\mathrm{n}^{\circ} 2002-102 \mathrm{du} 11$ février 2002, relative à la création, à la gestion et au financement des parcs nationaux et réserves naturelles qui sont des zones de conservation de la diversité biologiques. Les écosystèmes sont également régis par la loi $\mathrm{n}^{\circ}$ 96-766 du 03 Octobre 1996 portant code de l'environnement qui a pour objet la protection et la sauvegarde de l'environnement, dans la perspective d'un développement durable.

A priori, rien ne s'oppose donc au plan juridique à la protection de la diversité biologique et par ricochet à la protection des écosystèmes et des habitats. Mais, si la protection des écosystèmes et des habitats peut paraître aisées dans les aires protégées, elle apparaît difficile dans le domaine rural ou la sécurité foncière n'est pas maîtrisée (Ministère de l'Agriculture et du Développement Rural, 2017). Les détenteurs de droit coutumier sur terres forestières mènent à grand échelle des activités de conversion des forêts rurales en espaces agricoles (Kassoum, 2018). Cette pratique met en danger la survie de plusieurs espèces animales et végétales dans nos différentes régions. Aujourd'hui, pour inverser les tendances, l'état de Côte d'Ivoire à travers la Direction de l'Ecologie et de la Protection de la Nature (DEPN), département du Ministère de la Salubrité, de l'Environnement et du Développement exhorte les populations à faire de la conservation des ressources naturelles une priorité dans leur quotidien. Ce qui revient à inculquer aux populations un esprit de volontariat dans la création de réserves naturelles. C'est dans ce cadre que l'ONG CEM (Conservation des Espèces Marines) initie en collaboration avec les populations de Grand-Béréby un projet de création de Réserve Naturelle Volontaire (RNV) en vue de sauvegarder les grands mammifères en voie de disparition comme le chimpanzé d'Afrique de l'Ouest, Pan troglodytes verus (Bitty et al., 2013). On désigne par Réserve Naturelle Volontaire (RNV), une réserve naturelle partielle créée à l'initiative d'une collectivité territoriale, d'un établissement public ou d'une personne de droit privé, sur un terrain lui appartenant et pour la préservation d'un écosystème ou d'un paysage remarquable (Journal officiel de Côte d'Ivoire, 2002). En d'autres termes, les RNV sont des domaines privés qui présentent un intérêt particulier sur les plans scientifique, écologique et touristique. Ces réserves privées ont pour rôle d'une part de contribuer à la conservation de la diversité biologique du 
pays, et de lutter contre la dégradation de l'environnement naturel (désertification, déforestation, dégradation des sols etc.) et d'autre part de contribuer à l'amélioration du microclimat, à la perpétuation des mythes culturels des localités par la protection de certaines espèces et la mise à disposition de certains produits forestiers.

L'étude de la flore et de la végétation pour évaluer la valeur pour la conservation d'une forêt constitue une étape importante pour donner le statut de RNV. Plusieurs cas d'études menées dans ce sens existent dans la littérature. L'on peut citer celle de la Fondation IGF (2007), pour donner un statut de RNV à la Réserve Naturelle de Faune de N'Zi River Lodge, celle de Vroh et al. (2010) pour la création de la RNV à Azaguié, celle de Zadou (2011) pour la conversion de la Forêt des Marais Tanoé-Ehy en une RNV, celle de la compagnie fruitière SCB (2012) pour la protection d'une forêt sur son terrain ou encore celle de Adou Yao et al. (2013) dans le sens de la conversion de la Forêt Sacrée Bokasso en une RNV. Il s'avère donc nécessaire de déterminer le caractère particulier de la RNV de la Dodo. C'est en cela que cette étude trouve toute sa justification. Elle vise à montrer la valeur pour la conservation de la Réserve Naturelle Volontaire de la Dodo. De façon spécifique, il s'est agi de décrire la végétation et la flore de la RNV, d'évaluer la diversité de la flore et de déterminer les espèces végétales à statut particulier, notamment les espèces rares, les espèces en voie de disparition ou encore, les espèces endémiques.

\section{MATERIEL ET METHODES \\ Site d'étude}

La Réserve Naturelle Volontaire qui a fait l'objet d'étude est une forêt côtière située dans le domaine rural des villages de Manibereby et Pitiké ; deux villages de la souspréfecture de Grand-béréby (Figure 1). Sa superficie est estimée à 4694 hectares. Elle est limitée au Sud par la mer, à l'est par la rivière Dodo, à l'ouest par les villages de Pitiké et Tabéko et au nord par le domaine de la SoGB (Société des caoutchoucs de Grand-Béréby).

\section{Méthode de collecte de données}

Deux techniques de relevés de terrain ont été utilisées. Il s'agit du relevé de surface et du relevé itinérant. Ces deux types de relevés sont complémentaires. Le relevé de surface a consisté à délimiter le long de chaque transect préalablement établis pour l'observation des animaux une parcelle de 400 $\mathrm{m}^{2}(40 \mathrm{~m} \times 10 \mathrm{~m})$ comme présenté sur la

Figure 2. A l'intérieur de cette surface, toutes les espèces de plantes rencontrées sont identifiées et leurs noms scientifiques sont notés sur la fiche de relevé portant le numéro du transect. Ces placettes ont été disposées en début de transect, au milieu et à $40 \mathrm{~m}$ de la fin du transect en allant dans le sens Est-Ouest.

Des relevés itinérants ont été réalisés dans tous les espaces visités. Ce relevé n'a concerné que les espèces non rencontrées dans les relevés de surface. Les espèces observées, dans ce deuxième type d'inventaire, sont notées et des échantillons récoltés pour compléter la liste floristique générale.

\section{Méthode d'analyse de données Richesse et composition floristique}

Le nombre total d'espèces inventoriées pour les différents types de végétation a été déterminé. Pour chacune des espèces inventoriées, nous avons ensuite noté la famille, le genre, les types biologiques et la répartition phytogéographique.

\section{Diversité spécifique}

Elle a été estimée à partir des indices de diversité de Shannon $\left(H^{\prime}\right)$ et d'équitabilité $(E)$ de Pielou. Ces deux indices ont été calculés selon les formules mathématiques suivantes :

\section{$H^{\prime}=-\Sigma(n i / N) \ln (\mathbf{n i} / \mathbf{N})$}

Dans cette formule, $H^{\prime}$ est l'indice de Shannon, ni le nombre d'individus d'une espèce $\mathrm{i}$ et $\mathrm{N}$ le nombre total d'individus de toutes les espèces.

Cet indice varie de 0 (une seule espèce présente) à $\ln S$ (toutes les espèces présentes ont la même abondance). Pour les communautés très diversifiées, $H^{\prime}$ peut atteindre 4,5 (Kenth et Coker, 1992) et $\ln S$ dépasse rarement 5 (Felfili et al., 2004).

$$
E=H^{\prime} / \operatorname{lnS}
$$

Où $\mathrm{E}$ est l'indice d'équitabilité de Piélou et $\mathrm{S}$ le nombre total d'espèces d'un biotope. 
L'équitabilité varie de 0 à 1 . Elle tend vers 0 quand la quasi-totalité des effectifs est concentrée sur une espèce et vers 1 lorsque toutes les espèces ont la même abondance. Dans le cas où cet indice tend vers 1 , le milieu en question est dit équilibré.

\section{Valeur de conservation}

D'une part la valeur pour la conservation la réserve a été estimée et d'autre part, ses biotopes ont été évalués individuellement afin de déceler les biotopes prioritaires pour la conservation. Ces évaluations ont été faites en se rapportant à la présence d'espèces à statut particulier pour ce qui est de la première Haute Valeur de Conservation (HVC 1) prônée par la Forest Standarship Council (FSC). Il s'agit dans un premier temps des espèces endémiques sur la base des listes d'espèces préétablies par AkéAssi (2001; 2002), Pooter et al. (2004) et dans un second temps des espèces rares et/ou menacées d'extinction aussi bien sur le plan international que national selon l'UICN (2015) et Aké-Assi (1998). En ce qui concerne l'endémisme, nous avons distingué les endémiques aux blocs forestiers Ouest africains (GCW) parmi lesquelles celles qui sont propres au territoire ivoirien sont désignées par GCi (Aké-Assi, 2001 ; 2002). Les espèces endémiques aux forêts de la région phytogéographique de la Haute Guinée sont désignées par HG. Le dernier critère évalué est la présence d'habitats particuliers pouvant être répertoriés HVC3, caractéristique des écosystèmes, habitats ou zones refuges rares, menacés ou en voie de disparition.

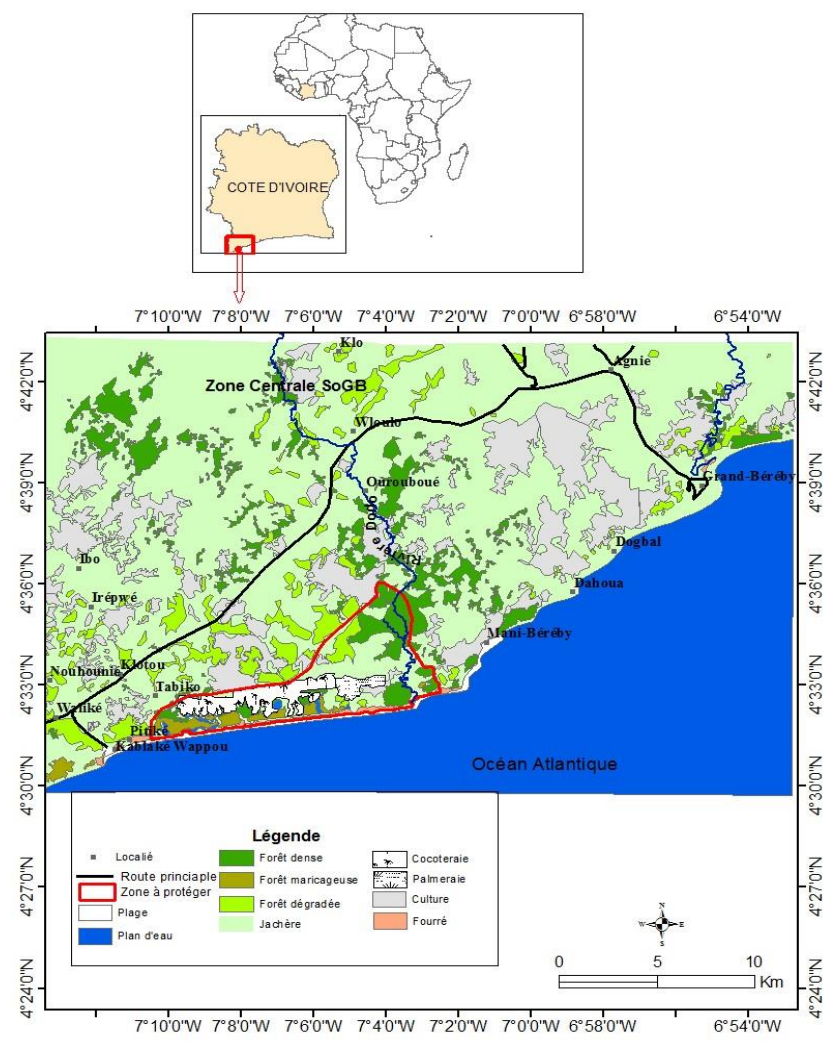

Figure 1 : Situation géographique de la Réserve Naturelle Volontaire (RNV) de la Dodo.

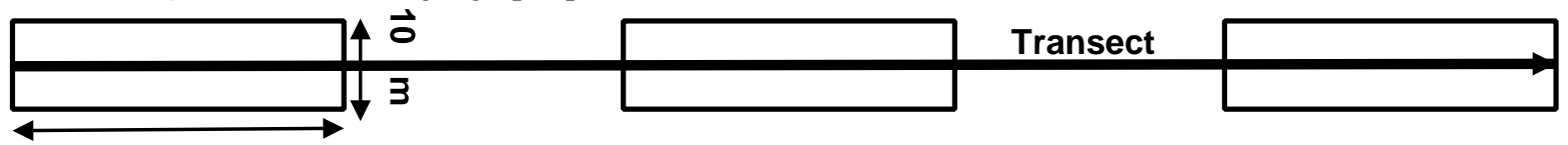

$40 \mathrm{~m}$

Figure 2 : Illustration de la disposition des placettes sur les transects d'observation de la présence des animaux. 


\section{RESULTATS \\ Caractéristiques de la végétation}

Dans la Réserve Naturelle Volontaire, les différents types de végétation rencontrés sont les jachères, les savanes incluses, les forêts denses, les forêts secondaires et les forêts galeries.

Les jachères sont des anciens champs de vivriers et des plantations de palmiers à huile non entretenues. Ces espaces sont envahis par des espèces héliophiles et plusieurs autres espèces adaptées aux milieux perturbés. On y rencontre, en abondance, les espèces telles que Harungana madagascariensis Lam. ex Poir., Trema guineensis (Schum. \& Thonn.) Ficalho, Aframomum daniellii (Hook.f.) K. Schum, Musanga cecropioides R. Br., Alchornea cordifolia (Schum. \& Thonn.) Müll.Arg., Hymenostegia afzelii (Oliv.) Harms, Pterocarpus santalinoides L'Hérit. ex DC., Manotes longiflora Baker, Chromolaena odorata (L.) R. M. King \& H. Rob.

Les savanes incluses sont présentes sur de grandes superficies. Elles sont continuellement fréquentées par les grands mammifères herbivores. Dans ces espaces les espèces dominantes sont Chrysobalanus icaco Linn.subsp. Icaco, Ochna multiflora DC., Martretia quadricornis Beille, Xylopia parviflora (A. Rich.) Benth., Melastomastrum capitatum (Vahl) A. \& R. Fern., Tricalysia macrophylla K. Schum, Dactyladenia barteri, Pterocarpus santalinoides L'Hérit. ex DC.

Les forêts denses, quant à elles, sont des forêts érigées pour la plupart sur des sols hydromorphes. Elles sont inondées en saison des pluies. Ce qui rend leurs accès très difficile et par ricochet contribue à leur conservation. Les espèces les plus rencontrées dans ces milieux sont Cola reticulata A.

Chev., Hunteria eburnea Pichon, Xylopia rubescens Oliv., Trichoscypha arborea (A. Chev.) A. Chev., Mitragyna ledermannii (K. Krause) Ridsdale, Carapa procera DC. De Wilde, Diospyros mannii Hiern,
Hymenostegia afzelii (Oliv.) Harms.

Les forêts secondaires sont des zones anciennement exploitées pour les grumes. Elles sont dominées par les espèces telles que Cephaëlis peduncularis Salisb. var. tabouensis (Schnell) Hepper, Dactyladenia barteri, Ouratea glaberrima (P. Beauv.) Engl. ex Gilg A, Xylopia parviflora (A. Rich.) Benth., Pentaclethra macrophylla Benth., Lophira alata Banks ex Gaertn.f., Ancistrocladus barteri Sc. Elliot, Cola reticulata A. Chev.

Les forêts galeries sont situées le long de la rivière Dodo. Ce sont des milieux dominés par les espèces de raphia et de rotin telles que Raphia hookerii, Calamus deeratus, Phoenix reclinata, Lygodium microphyllum. Ces milieux sont les plus perturbés à cause de l'approvisionnement régulier en palmes par les populations des campements riverains. Les palmes coupés servent à la construction de maisons.

\section{Richesse et composition floristique}

Dans la RNV, il a été inventorié 242 espèces reparties en 193 genres appartenant à 70 familles. Les genres les plus diversifiés sont Ficus (8 espèces), Diospyros (6 espèces), Xylopia (6 espèces), Solanum (4 espèces) et Strychnos (4 espèces). Les familles les plus diversifiées par ordre d'importance comme l'indique la Figure 3 sont les Euphorbiaceae (7,44\% des espèces), les Rubiaceae (5,79\%), les Caesalpiniaceae $(5,37 \%)$, les Annonaceae $(4,96 \%)$, les Apocynaceae (4,55\%), les Moraceae $(4,55 \%)$, les Fabaceae $(4,13 \%)$ et les Poaceae $(3,72 \%)$.

Les espèces se rangent en 10 types biologiques. Les espèces microphanérophytes (mp) qui représentent $45,19 \%$ de l'ensemble sont les plus abondantes. Elles sont suivies par les mésophanérophytes (mP), les nanophanérophytes (np) et les mégaphanérophytes. Ces espèces représentent respectivement $23,43 \%, 14,23 \%$ et $5,86 \%$ de l'ensemble des espèces (Figure 4). 
En ce qui concerne la répartition phytogéographique des espèces, on note que celles de la région Guinéo-Congolaise (GC) sont les plus nombreuses (Figure 5). Elles représentent $70,71 \%$ de l'ensemble. Les espèces les moins représentées sont celles de la région Soudano-Zambézienne (SZ) qui représente $0,84 \%$ de l'ensemble. Les autres groupes restent intermédiaires à ces deux valeurs extrêmes.

\section{Diversité spécifique}

Dans l'ensemble, les forêts denses et les jachères rencontrées dans la RNV ont des diversités plus élevées (Tableau 1). Ce sont les savanes incluses qui sont les moins diversifiées $(H=2,45)$. L'indice d'équitabilité reste élevé pour l'ensemble des biotopes. Ce qui signifie que les espèces sont équitablement réparties dans tous les biotopes.

\section{Valeur de conservation}

La RNV constitue un refuge d'espèces à statut particulier. Le nombre de ces espèces est estimé à trente (30). Parmi celles-ci, on dénombre 21 espèces endémiques dont une (1) endémique à la Côte d'Ivoire à savoir Baphia bancoensis Aubrév, cinq (5) à la Haute Guinée (HG) et cinq (5) au bloc forestier à l'ouest du Togo, comprenant le Ghana, la Côte d'Ivoire, le Libéria, la Sierra Léone, la Guinée, la Guinée Bissau, la Gambie et le Sénégal $(\mathrm{GCW})$. Seize (16) espèces sont à la fois de la haute guinée et du bloc forestier à l'ouest du Togo (Tableau 2). On estime au nombre de quatorze (14) les espèces rares et/ou menacées d'extinction selon UICN (2015) et Aké-Assi (1998). On dénombre deux (2) identifiées par Aké-Assi comme Plantes rares, devenues rares et en voies d'extinction (PRE). Cinq (5) autres sont classées par l'UICN comme des espèces vulnérables et trois (3) sont à la fois vulnérables selon l'UICN (2015) et Plantes rares, devenues rares et en voies d'extinction (PRE) selon Aké-Assi (1998). Dans ce dernier lot, on a les espèces telles que Garcinia afzelii Engl., Garcinia kola Heckel et Milicia regia A. Chev. L'espèce Pellegriniodendron diphylium (Harms) J. Léonard a pour statut risque faible/quasi menacé. C'est dans les forêts denses et forêts secondaires qu'on rencontre en abondance les espèces à statut particulier. Les savanes incluses en comptent moins.

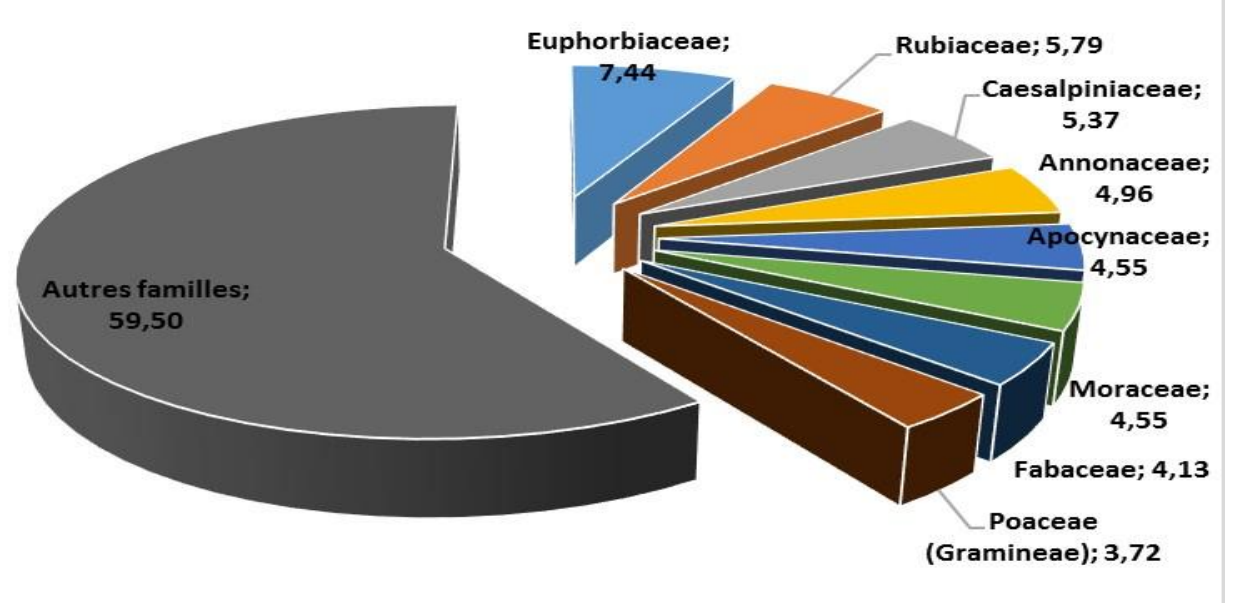

Figure 3: Spectre des familles des espèces inventoriées. 


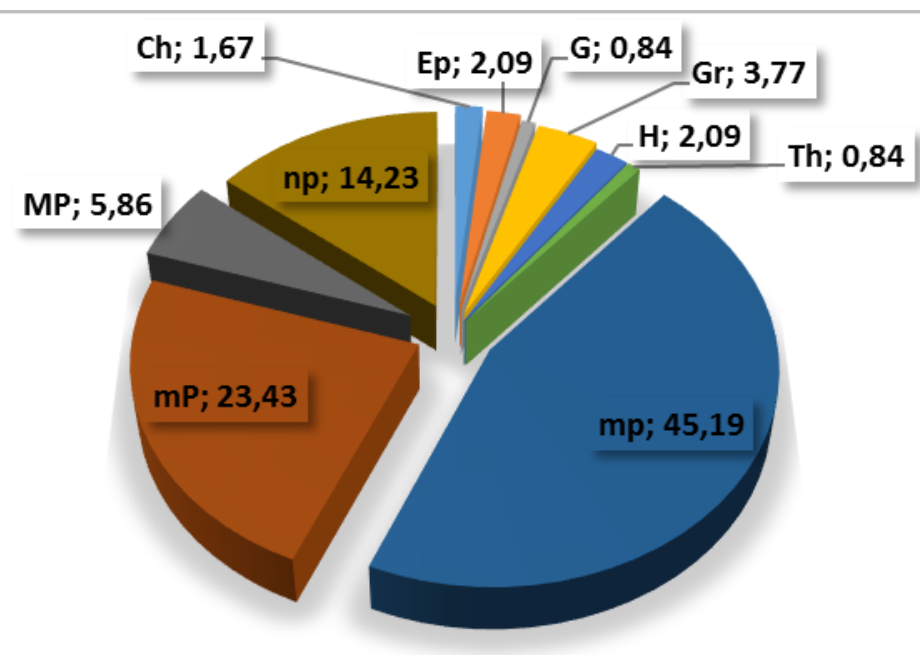

Figure 4 : Spectre des types biologiques des espèces.

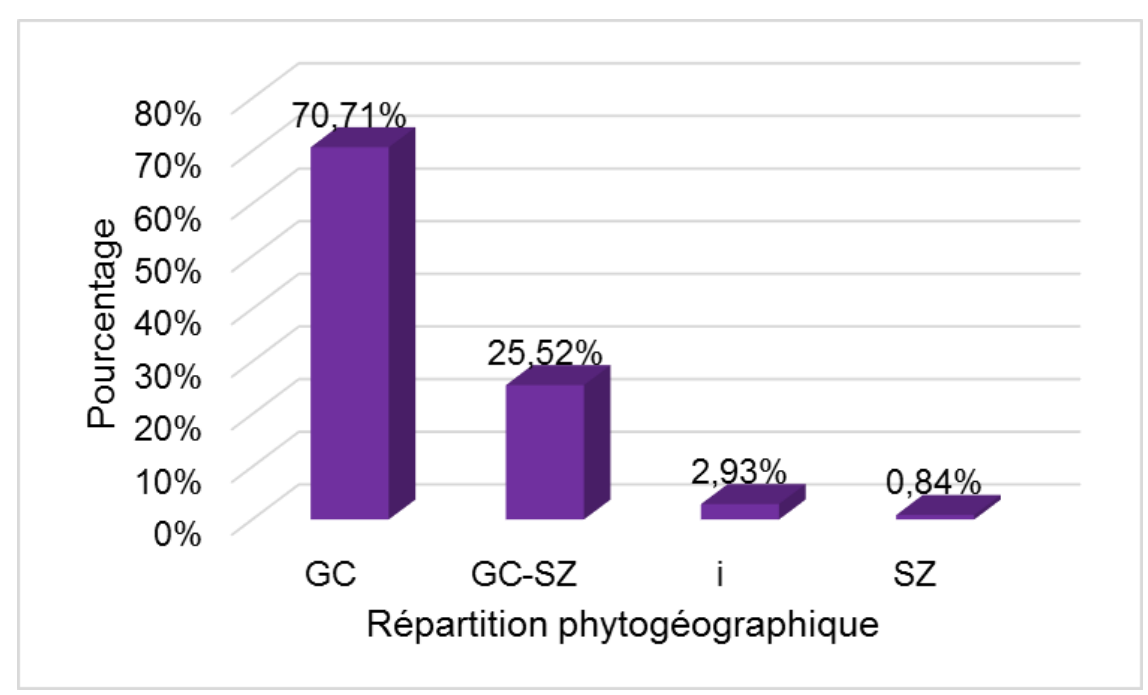

Figure 5: Répartition phytogéographique des espèces recensées.

Tableau 1: Indice de diversité des différents biotopes.

\begin{tabular}{lcc}
\hline Biotope & Indice de Shannon & Indice d'équitabilité \\
\hline Forêt dense & 3,008 & 0,713 \\
\hline Forêt secondaire & 2,578 & 0,603 \\
\hline Jachère & 3,506 & 0,817 \\
\hline Savane incluse & 2,455 & 0,753 \\
\hline
\end{tabular}


Tableau 2: Liste des espèces endémiques recensées dans la RNV.

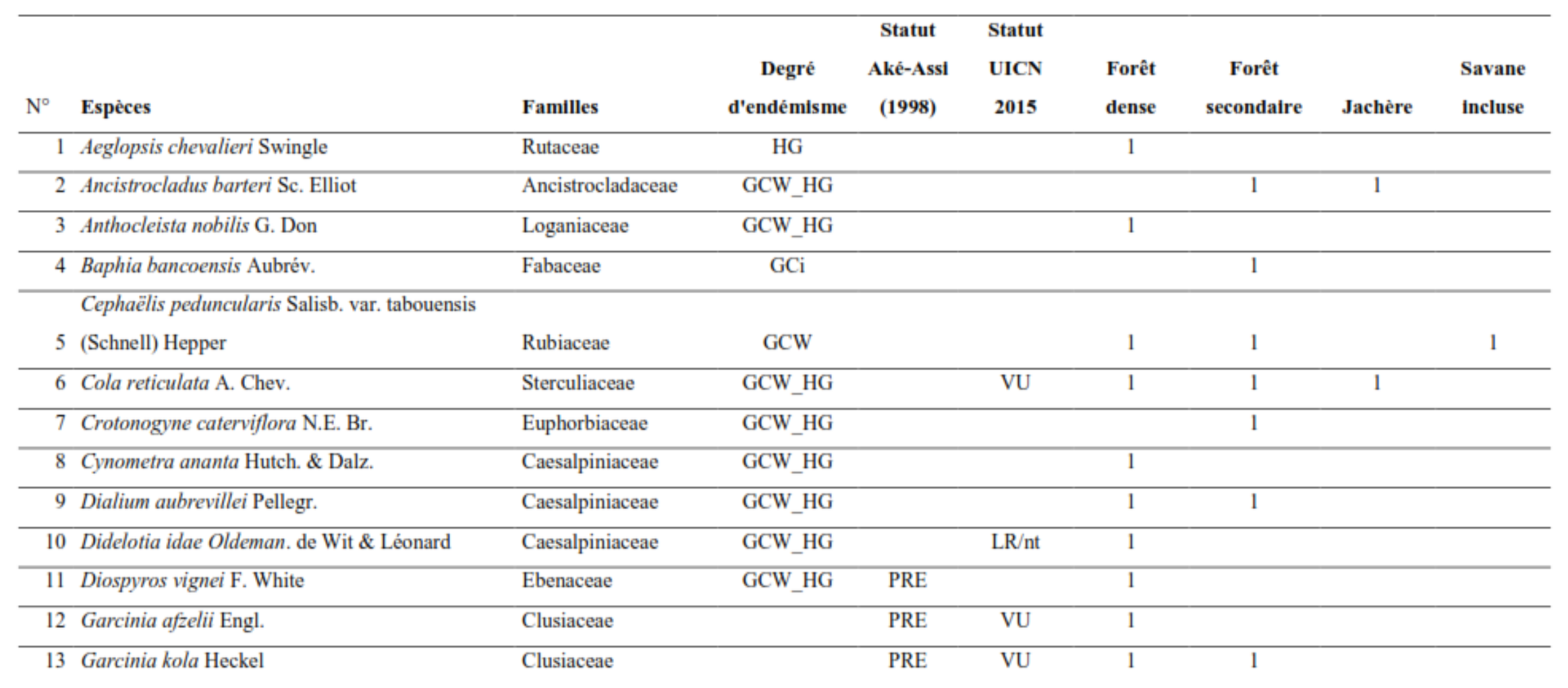


Y. J.-C. KOUADIO et al. / Int. J. Biol. Chem. Sci. 12(6): 2784-2796, 2018

\begin{tabular}{|c|c|c|c|c|c|c|c|c|}
\hline 14 Isomacrolobium vignei (Hoyle) Aubrév. Pellegr. & Caesalpiniaceae & GCW_HG & & vU & & & 1 & \\
\hline 15 Lannea nigritana (Sc. Elliot) Keay var. nigritana & Anacardiaceae & & PRE & & & 1 & 1 & \\
\hline 16 Lophira alata Banks ex Gaertn.f. & Ochnaceae & & & VU & & 1 & & \\
\hline 17 Maranthes aubrevillei (Pellegr.) France & Chrysobalanaceae & GCW_HG & & & & 1 & & \\
\hline 18 Memecylon afzelii var. amoenum Jac.-Fél. & Melastomataceae & GCW & & & 1 & & & \\
\hline 19 Milicia regia A. Chev. & Moraceae & GCW_HG & PRE & VU & & & 1 & \\
\hline 20 Millettia rhodontha Baill. & Fabaceae & GCW_HG & & & & 1 & & \\
\hline 21 Mitragyna ledermannii (K. Krause) Ridsdale & Rubiaceae & & & VU & 1 & & & \\
\hline 22 Nauclea diderrichii (De Wild.\& T. Durand) Merr. & Rubiaceae & & & VU & & 1 & & \\
\hline 23 Panicum repens Linn & Poaceae (Gramineae) & & & LC & & & & 1 \\
\hline Pellegriniodendron diphylium (Harms) J. & & & & & & & & \\
\hline 24 Léonard & Caesalpiniaceae & & & $\mathrm{LR} / \mathrm{nt}$ & 1 & & & 1 \\
\hline 25 Pterocarpus santalinoides L'Hérit. ex DC. & Fabaceae & & & $\mathrm{LR} / \mathrm{nt}$ & & 1 & 1 & 1 \\
\hline 26 Ptychopetalum anceps Oliv. & Olacaceae & HG & & & & 1 & & \\
\hline Scytopetalum tieghemii A. Chev. ex Hutch. \& & & & & & & & & \\
\hline 27 Dalziel & Scytopetalaceae & GCW_HG & & & 1 & & & \\
\hline 28 Strychnos dinklagei Gilg & Loganiaceae & GCW_HG & & & 1 & & & \\
\hline 29 Tiliacora dinklagei Engl. & Mennispermaceae & GCW & & & 1 & 1 & 1 & \\
\hline 30 Urera rigida (Benth.) Keay & Urticaceae & HG & & & & & 1 & \\
\hline Total & & & & & 16 & 15 & 8 & 4 \\
\hline
\end{tabular}

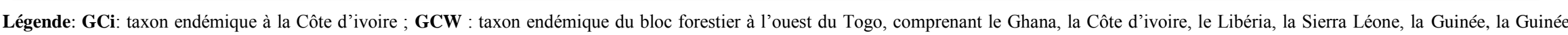

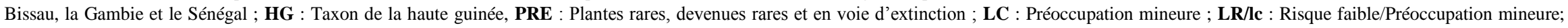

LR/nt : Risque faible/Quasi menacé VU : Vulnérable. 


\section{DISCUSSION}

La désignation de la forêt de la Dodo, objet de cette étude relève de l'observation faite par des primatologues sur la présence d'un petit groupe de chimpanzé (Pan troglodytes verus) dans cette forêt. Cette étude voudrait apporter des preuves supplémentaires sur le plan botanique de sa Haute Valeur de Conservation (HVC). Il y a six catégories de HVC qui recouvrent des valeurs à la fois écologiques et sociales. Une évaluation complète des Forêts de Haute Valeur de Conservation (FHVC) doit tenir compte des six valeurs. Dans cette étude, il a été procédé à une évaluation partielle, focalisée, sur les valeurs écologiques comme il est parfois utile de le faire. Ainsi, l'évaluation de la valeur de conservation de la RNV à travers la diversité des espèces (HVC 1) et la particularité des écosystèmes et habitats (HVC 3 ) a été faite.

L'étude a permis de recenser 242 espèces végétales. Cette richesse floristique semble plus élevée que celle observée par les auteurs Vroh et al. (2010) sur le site d'une Réserve Naturelle Volontaire à Azaguié, SudEst de la Côte d'Ivoire. Cette différence pourrait s'expliquer dans un premier temps par la différence de zones écologiques et dans un second temps par la technique de collecte de données. En effet, la position géographique de la Reserve Naturelle Volontaire d'Azaguié (Sud-Est) diffère de celle de la Réserve Naturelle Volontaire de la Dodo qui se situe au Sud-Ouest de la Côte d'Ivoire. Aussi, Vroh et al. (2010) ont-ils considéré uniquement les individus arborescents dont le diamètre à hauteur de poitrine (DBH) atteint un minimum de $2,5 \mathrm{~cm}$ tandis que dans cette étude tous les individus sans distinction de DBH ont été pris en compte. Le nombre élevé d'espèces recensées est loin de refléter toute la réalité, compte tenu de la faible surface échantillonnée. En effet, on y rencontre dans la réserve des espaces marécageux difficiles d'accès en saison des pluies, la saison de la campagne d'inventaire. Des campagnes d'inventaires pourraient se réaliser dans ces espaces en saison sèche pour permettre d'avoir une liste plus exhaustive.

La dominance des Euphorbiaceae, Rubiaceae, Caesalpiniaceae, Annonaceae,
Apocynaceae, Moraceae et Fabaceae n'est pas spécifique à la RNV de la Dodo. Ce même cortège floristique a déjà été signalé dans la plupart des forêts ivoiriennes (Nusbaumer et al., 2005 ; N'Da et al., 2008, Kouamé et al., 2010). La RNV de la Dodo serait une forêt qui n'a pas encore atteint le stade climacique vu l'abondance des Rubiaceae (Adou Yao, 2005, Tuo et al., 2017).

La dominance des espèces de la région Guinéo-Congolaise (GC) est une preuve que cette zone appartient bien à la région GuinéoCongolaise (Tiokeng, 2015). La proportion des espèces Guinéo-Congolaise qui tend vers $80 \%$ confirme l'assertion de Solefack (2018) qui soutient que la flore de cette région phytogéographique doit avoir plus de 80 à $90 \%$ d'endémiques et seulement environ $10 \%$ d'espèces d'autres régions phytogéographiques. Cette dominance témoigne également du niveau de conservation élevé du site. La flore n'a donc pas perdu sa spécificité malgré la présence de traces d'anthropisation, de cultures et de jachères.

La RNV constitue un refuge d'espèces à statut particulier en ce sens qu'elle regorge une concentration d'espèces vulnérables, en danger ou menacées d'extinction, ainsi que des espèces endémiques. On estime à trente (30) le nombre de ces espèces. Cette caractéristique de la RNV lui confère la première Haute Valeur de Conservation (HVC 1) qui exigence une concentration de diversité biologique incluant les espèces endémiques et les espèces rares, menacées ou en danger importantes à l'échelle internationale, régionale ou nationale.

Par ailleurs, l'endémisme est considéré comme un critère important pour évaluer la priorité et l'état de conservation d'un espace donné. La présence d'espèces endémiques témoigne d'un milieu conservé (Adou Yao, 2005). A l'opposé, leur absence est synonyme d'un milieu dégradé car elles sont hautement vulnérables à la perturbation humaine et aux autres formes de changements environnementaux. La présence en grand nombre de ces plantes est également signe d'une grande biodiversité. Par conséquent, leur distribution est fréquemment utilisée pour indiquer des 'Hotspots' de biodiversité (Myers, 2000). 
En considérant les types de biotopes, on constate que les forêts denses et les jachères sont les plus diversifiées devant les forêts secondaires. Par contre, ce sont les forêts denses et forêts secondaires qui comptent le plus d'espèces à statut particulier. Ce résultat vient conforter l'assertion de Ricotta (2000) selon laquelle la richesse spécifique et l'indice de Shannon seuls ne seraient pas adaptés à l'estimation de la valeur écologique d'un site. Par conséquent, elles ne pourraient permettre l'identification des Hautes Valeurs de Conservation d'une concession étudiée. En effet, la richesse spécifique caractérisant les jachères n'est pas de nature forestière, est instable et comporte peu d'espèces de haute valeur patrimoniale (De Warnaffe, 2001). Elle ne décrit que l'aspect composition taxonomique, d'une manière synthétique et approximative. Elle a certes un intérêt patrimonial, mais pas nécessairement écologique. Quant à l'indice de Shannon, il est assez insensible à la présence d'espèces rares nécessaire à l'identification et la gestion des HVC. Ainsi, en considérant la présence d'espèce à statut particulier dans les types de biotopes, les forêts denses et les forêts secondaires de la RNV de la Dodo constituent des zones refuges de biodiversité, une caractéristique exigée dans l'identification et la gestion des Hautes Valeurs de Conservation de catégorie 3 (Daïnou et al., 2017). Dans ce sens, les forêts denses et les forêts secondaires de la RNV pourraient être répertoriés comme des HVC de catégorie 3 .

La RNV dipose de vaste étendues de savanes incluses qui representent des habitats particuliers que l'on retrouve uniquement dans le paysage côtier de la Côte d'Ivoire. Ce sont des écosystèmes rares, menacés ou en voie de disparition à cause des activités anthropiques croissantes. Leur rareté et leur particularité permettent de les identifier comme des Hautes Valeurs de Conservation de la catégorie 3.

En somme, la RNV de la Dodo renferme les valeurs $\mathrm{HVC} 1$ et $\mathrm{HVC} 2$ donc dispose d'une valeur pour la conservation élevée. Il est primordial que des mesures pour sa gestion durable soit élaborées car ces genres d'écosystèmes, s'ils restent sans mesure de protection, sont vulnérables à la déforestation au profit des activités agricoles ou à la fragmentation forestière (Pooter et al., 2004 ; Bakayoko, 2005). Suivant le principe $9 \mathrm{du}$ FSC toutes activités de gestion durable de la RNV devront conserver ou augmenter les attributs qui la caractérisent.

\section{Conclusion}

L'étude conduite dans la Réserve Naturelle Volontaire de la Dodo a contribué à une meilleure connaissance de sa valeur de conservation à travers les caractéristiques de sa végétation et de sa composition floristique. Il ressort de cette étude que les différents types de végétation rencontrés sont les jachères, les cultures pérennes (palmeraies, cocoteraies), les forêts denses, les mangroves, les forêts galeries et les savanes incluses. Il a été inventorié 242 espèces reparties 193 genres appartenant à 70 familles. Les genres les plus diversifiés sont Ficus, Diospyros, Xylopia, Solanum et Strychnos. Les familles les plus diversifiées par ordre d'importance sont les Euphorbiaceae, les Rubiaceae, les Caesalpiniaceae, les Annonaceae, les Apocynaceae, les Moraceae, les Fabaceae et les poaceae. Les espèces se rangent en 10 types biologiques. Les espèces de la région Guinéo-Congolaise (GC) sont les plus nombreuses. La Réserve Naturelle Volontaire constitue un refuge d'espèces à statut particulier. Le nombre de ces espèces est estimé à trente (30). Parmi celles-ci, on dénombre 21 espèces endémiques et 14 les espèces rares et/ou menacées d'extinction selon UICN (2015) et Aké-Assi (1998). Les forêts denses et les jachères rencontrées dans la RNV ont des diversités plus élevées. Ce sont les savanes incluses qui sont les moins diversifiées. La RNV dispose les valeurs de conservation HVC1 et HVC3. Une étude écologique plus approfondie permettrait de mettre en évidence d'autres catégories de HVC qu'elle regorgerait.

\section{CONFLIT D'INTERETS}

Les auteurs déclarent qu'il n'existe aucun conflit d'intérêts par rapport à cet article. 


\section{CONTRIBUTIONS DES AUTEURS}

YJCK est le principal investigateur de ce travail et a participé à toutes les phases du travail ; KSPS, WBK et NOY ont contribué à la collecte des données sur le terrain et à la rédaction de l'article; KEN, KPK et PJG ont contribué par leur lecture à l'amélioration et à la validation du manuscrit.

\section{REMERCIEMENTS}

Nous sommes très reconnaissants à l'ONG locale Conservation des Espèces Marines (CEM) pour son soutien technique, matériel et humain. Nous ne saurions oublier nos fidèles assistants et guides qui nous ont aidés à collecter des données de terrain au fil des jours.

\section{REFERENCES}

Adou Yao CY. 2005. - Pratiques paysannes et dynamiques de la biodiversité dans la forêt classée de Monogaga (Côte d'Ivoire), Thèse de Doctorat unique, département Hommes Natures Sociétés, MNHN, Paris (France), 248 p.

Adou Yao CY, Kpangui KB, Kouao KJ, Adou LMD, Vroh BTA, N'Guessan KE. 2013.

Diversité floristique et valeur de la forêt sacrée Bokasso (Est de la Côte d'Ivoire) pour la conservation. VertigO, 13 (1). id.erudit.org/iderudit/1026585ar

Aké Assi L. 2001. Flore de la Côte d'Ivoire 1, catalogue, systématique, biogéographie et écologie. Genève, Suisse : Conservatoire et Jardin Botanique de Genève ; Boisseria, 57, 396 p.

Aké Assi L. 2002. Flore de la Côte d'Ivoire 2, catalogue, systématique, biogéographie et écologie. Genève, Suisse :

Conservatoire et Jardin Botanique de Genève ; Boisseria, 58; 441 p.

Aké-Assi L. 1998. Impact de l'exploitation forestière et du développement agricole sur la conservation de la biodiversité biologique en Côte d'Ivoire. Le Flamboyant, 46 : 20-21.

Bakayoko A. 2005. Influence de la fragmentation forestière sur la composition floristique et la structure de la végétale dans le sud-ouest de la Côte
d'Ivoire. Thèse de doctorat, Université de Cocody-Abidjan, 258 p.

Bittty EA, Kadjo B, Bi SG, Okon MO,

Kouassi PK. 2013. Inventaire de la faune mammalogique d'une forêt urbaine, le Parc National du Banco, Côte d'Ivoire. International Journal of Biological and Chemical Sciences, 7(4): 1678-1687. DOI :

http://dx.doi.org/10.4314/ijbcs.v7i4.23

Daïnou K, Dubart N, Bracke C, Doucet J-L. 2017. Evaluation de la présence de hautes valeurs de conservation (HVC) dans les concessions gérées par la Société Pallisco, Cameroun : Identification, menaces potentielles et mesures de gestion des HVC, Université de Liège, 43p.

De Warnaffe GDB. 2001. Impact de la gestion forestière sur la biocénose en Région Wallonne. Convention MRW-UCL, 6e rapport. Unité des Eaux et Forêts, Faculté des Sciences Agronomiques, UCL. Louvain-la-Neuve, Belgique, 93p

Felfili JM, Silva Júnior MC, Sevilha AC, Fagg CW, Walter BMT, Nogueira PE, Rezende AV. 2004. Diversity floristic and structural patterns of cerrado vegetation in Central Brazil. Plant Ecology, 175: 37 - 46.

Fondation IGF. 2007. Réserve Naturelle de faune de N'Zo River Lodge, Côte d'Ivoire- Etudes préalables. Fondation IGF, $52 \mathrm{p}$.

Journal Officiel de Côte d'Ivoire. 2002. Loi $\mathrm{n}^{\circ}$ 2002-102 du 11-02-2002 relative à la création, à la gestion et au financement des parcs nationaux et réserves, Abidjan, Côte d'Ivoire, $26 \mathrm{p}$.

Kassoum T. 2018. Le couvert forestier en Côte d'Ivoire : une analyse critique de la situation de gestion des forêts (classées, parcs et réserves). The International Journal of Social Sciences and Humanities Invention, 5(02): 4387-4397. DOI: $10.18535 / \mathrm{ijsshi} / \mathrm{v} 5 \mathrm{i} 2.02$.

Kenth M, Coker P. 1996. Vegetation Description and Analysis: a Practical Approach. Belhaven Press: New York, John Wiley; 363 p. 
Kouamé D, Yao CYA, Nandjui A, N'Guessan EK. 2010. Le rôle de l'éléphant dans la germination des graines de Irvingia gabonensis (Irvingiaceae), Balanites wilsoniana (Balanitaceae), Parinari excelsa (Chrysobalanaceae) et Sacoglottis gabonensis (Humiriaceae) en forêt tropicale: cas du Parc National d'Azagni. International Journal of Biological and Chemical Sciences, 4(5): 1442-1454. DOI : http://ajol.info/index.php/ijbcs

Ministère de l'Agriculture et du Développement Rural. 2017. Déclaration de politique foncière rurale de la Côte d'Ivoire. Déclaration de Politique Foncière Rurale de la Côte d'Ivoire, $31 \mathrm{p}$

Myers N, Mittermeier RA, Mittermeier CG, Da Fonseca GAB, Kent J. 2000. Biodiversity hotspots for conservation priorities. Nature, 403: 853-858. DOI : 10.1038/35002501

N'Da DH, Adou Yao CY, N'Guessan KE, Koné M, Sangne YC. 2008. Analyse de la diversité floristique du Parc National de la Marahoué, Centre-ouest de la Côte d'Ivoire. Afrique SCIENCE, 4(3) : 552579.

Nusbaumer L, Gautier L, Chatelain C, Spichiger R. 2005. Structure et composition floristique de la forêt classée de la Scio (Côte d'Ivoire). Etude descriptive et comparative. Candollea, 60(2): 393-443.

Poorter L, Bongers F, Kouamé FN, Hawthorne WD. 2004. Biodiversity of West African Forests: An Ecological Atlas of Woody Plant Species. CABI Publishing, Nederland, Pays-Bas, $521 \mathrm{p}$.

Ricotta C. 2002. Bridging the gap between ecological diversity indices and measures of biodiversity with Shannon's entropy: comment to Izsak and Papp. Ecological Modelling, 152 : 1-3.

https://doi.org/10.1016/S03043800(01)00 468-9.
SCB 2012. Responsabilité sociale et environnement. Rapport d'activité. SCB premimum, Abidjan, Côte d'Ivoire, $60 \mathrm{p}$.

Solefack MCM, Temgoua LF, Fedoung E, Djouba RZ. 2018. Végétation et spectres fonctionnels de la galerie forestière de Koupa Matapit (Ouest-Cameroun). GeoEco-Trop, 42(1), 147-158.

Tiokeng B, Mapongmetsem P, Nguetsop VF, Tacham WN. 2015. Biodiversité floristique et régénération naturelle sur les Hautes Terre de Lebialem (Ouest Cameroun). International Journal of Biological and Chemical Sciences, 9(1): 56-68. DOI : http://dx.doi.org/10.4314/ijbcs.v9i1.6

Tuo, F. N., Koffi, K. J., Kouassi, A. F., Kone, M., Adama, B., \& Bogaert, J. (2017). Etude de la diversité, de l'endémisme et de la distribution spatiale des Rubiaceae de Côte d'Ivoire. International Journal of Biological and Chemical Sciences, 11(2): 777-797. DOI : http://dx.doi.org/10.4314/ijbcs.v11i2.20

UICN 2015. IUCN Red List of Threatened Species. www.iucnredlist.org.

Vroh BTA, Adou Yao CY, Kouamé D, N'Da DH, N'Guessan KE. 2010. Diversités Floristique et Structurale sur le Site d'une Réserve Naturelle Volontaire à Azaguié, Sud-est de la Côte d'Ivoire. European Journal of Scientific Research, 45 (3) : 411-421

Zadou DA. 2011. Valeur de la Forêt des Marais Tanoé-Ehy (Sud-Est de la Côte d'Ivoire) pour la conservation : dimension socio-anthropologique. Tropical Conservation Science, 4(4) : 373-385. Available online: www.tropicalconservationscience.org. 